\title{
Inflammatory markers in hospitalized patients with bacterial pneumonia: comparison of Gram-positive versus Gram-negative etiology and admission versus discharge findings
}

Hatice Turker, Armagan Hazar, Birsen Ocakli

Clinic of Chest Diseases, University of Health Sciences Süreyyapaşa Chest Diseases and Thoracic Surgery Training and Research Hospital, İstanbul, Turkey

Submitted: 4 October 2019

Accepted: 10 January 2020

Arch Med Sci

DOI: https://doi.org/10.5114/aoms.2020.95051

Copyright (c) 2020 Termedia \& Banach

\section{Abstract}

Introduction: The aim of the study was to evaluate inflammatory markers (neutrophil to lymphocyte ratio [NLR], platelet to lymphocyte ratio [PLR], platelet to mean platelet volume [PLT/MPV], C-reactive protein [CRP], CRP/ albumin) in hospitalized patients with bacterial community-acquired pneumonia (CAP) and to differentiate between Gram-positive and Gram-negative groups and between admission and discharge findings.

Material and methods: A total of 170 adult patients hospitalized with bacterial CAP due to Gram-positive $(n=130)$ and Gram-negative $(n=40)$ pathogens were included in this retrospective study. Complete blood count (CBC) and inflammatory markers (NLR, PLR, platelet/MPV ratio, CRP, albumin) were recorded.

Results: Gram-negative vs. Gram-positive pneumonia was associated with significantly higher likelihood of being hospitalized at the ICU $(70.0 \%$ vs. $2.3 \%$, $p<0.001)$, mortality $(20.0 \%$ vs. $2.3 \%, p<0.001)$ and sepsis $(27.5 \%$ vs. $0.0 \%$, $p<0.001)$. Neutrophil to lymphocyte ratio, PLR and PLT/MPV at admission or discharge showed no significant difference between study groups. A significant decrease in NLR $(p<0.001$ and $p=0.026$, respectively), CRP $(p<0.001$ and $p=0.013$, respectively) and CRP/albumin ratio ( $p=0.021$ and $p=0.037$, respectively) was noted from admission to discharge in both groups.

Conclusions: In conclusion, our findings in a retrospective cohort of hospitalized CAP patients revealed no significant role of NLR, PLR, PLT/MPV or CRP in differential diagnosis of Gram-negative versus Gram-positive etiology and thus no additional benefit of these markers in faster implementation of appropriate treatment in accordance with the Gram stain.

Key words: neutrophil to lymphocyte ratio, C-reactive protein (CRP), bacterial pneumonia, Gram-positive, Gram-negative.

\section{Introduction}

Community-acquired pneumonia (CAP) remains a major cause of death worldwide as it is associated with a hospitalization rate of $22-42 \%$ and mortality rate of $5-12 \%$, which rises to over $50 \%$ in intensive care unit (ICU) admissions [1-3].

Early diagnosis and immediate pathogen recognition are crucial in selection of an appropriate antibiotic regimen in pneumonia given that

\author{
Corresponding author: \\ Assoc. Prof. Birsen Ocakli \\ Clinic of Chest Diseases \\ University of Health Sciences \\ Süreyyapaşa Chest Diseases \\ and Thoracic Surgery Training \\ and Research Hospital \\ İstanbul, Turkey \\ E-mail: \\ birsenocakli@hotmail.com
}


treatment success increases as a function of the time of treatment initiation [4-6]. Accordingly, current pneumonia management guidelines emphasize rapid recognition of pathogens and early initiation of appropriate antimicrobial therapy to improve patient outcomes [7, 8]. However, the etiological diagnosis of bacterial pneumonia often remains uncertain due to delay and unreliable findings related to pathogen identification with current microbial diagnostic techniques in real-life clinical practice [5-10]. Although Gram staining is a widely accepted test with diagnostic and prognostic value and certain advantages such as being a readily available, inexpensive and rapid test without need of sophisticated equipment and no hazard to the patient $[6,9,11]$, controversy exists on the reliability of sputum Gram stain to guide initial antimicrobial treatment of CAP, with substantial variance in sensitivity and specificity in different settings [6, 10, 12, 13].

Hence, assessment of biomarkers has become an increasingly used approach to identify patients at risk, to determine severity and prognosis of disease and to guide initial antibiotic therapy [14-16]. Nonetheless, it should be noted that while sputum and blood cultures were primarily recommended in patients with severe disease in the 2007 ATS/IDSA Guideline, they are now recommended not only in patients with severe disease but also in all inpatients empirically treated for MRSA or Pseudomonas aeruginosa as per the 2019 ATS/IDSA Guideline [17]. In addition to clinical judgement, use of validated clinical scoring tools such as the Pneumonia Severity Index (PSI) and the CURB-65 (tool based on confusion, urea level, respiratory rate, blood pressure, and age $>65$ ) has also been recommended to determine the need for hospitalization in adults diagnosed with CAP [17].

The host inflammatory response in the pathogenesis of pneumonia has become the focus of recent research, and identifying inflammatory biomarkers with high sensitivity and specificity is considered likely to overcome treatment delays, to facilitate treatment decisions and to improve clinical severity assessment in patients with CAP $[5,18-21]$.

Alongside old markers such as white blood cell (WBC) count and C-reactive protein (CRP) level and neutrophil count [22], new inflammatory markers including the neutrophil to lymphocyte ratio (NLR) and the platelet to lymphocyte ratio (PLR) have received increasing scientific interest in the management of pneumonia [23, 24].

Both NLR and PLR are considered as novel markers predicting prognosis in several diseases including CAP $[18,20,25,26]$. Neutrophil to lymphocyte ratio has also been suggested to be a simple and even better marker in predicting bacteremia than WBC count and CRP level [19], to predict mortality and prognosis in CAP with a better profile than CRP and to reflect a more balanced inflammatory response and thus enable more precise assessment of severity than WBC [18, 24].

However, while the relationship between NLR, PLR and clinical outcomes as well as the value of NLR in differential diagnosis of bacterial vs. viral etiology have been investigated in patients with pneumonia in a limited number of studies [19, 23], no study to date has investigated the inflammatory markers with respect to admission and discharge period in bacterial pneumonia, specifically in Gram-positive versus Gram-negative etiology.

This study was therefore designed to evaluate inflammatory markers (NLR, PLR, platelet/MPV and (RP, CRP/albumin) in hospitalized patients with bacterial CAP by comparing Gram-positive vs. Gram-negative etiology and admission vs. discharge findings.

\section{Material and methods}

\section{Study population}

A total 170 adult patients hospitalized with bacterial CAP due to Gram-positive $(n=130)$ and Gram-negative $(n=40)$ pathogens were included in this retrospective study conducted at a tertiary care center between January 2016 and October 2017. Patients aged > 18 years and hospitalized with ICD code J10-J16 were included in the study, while those with co-morbid malignancy, collagen vascular disease or hematologic diseases likely to affect NLR were excluded.

The study was conducted in full accordance with the local Good Clinical Practice (GCP) guideline and current legislation, while permission was obtained from the University of Health Sciences, Sureyyapasa Chest Diseases and Thoracic Surgery Training and Research Hospital Ethics Committee for the use of patient data for publication purposes (date of approval/protocol no: 02.05.2018/07-031).

\section{Data collection}

Data on patient demographics, Gram stain and type of bacterial pathogen, co-morbid disorders, CURB-65 scores, hospitalization and discharge dates, length of hospital stay (LOS), hospitalization unit (ICU, ward), in-hospital mortality and laboratory findings including complete blood count (CBC) and inflammatory markers including NLR, PLR, platelet/mean platelet volume (MPV) ratio and CRP were recorded. Findings on CBC and inflammatory markers were compared between Gram-positive vs. Gram-negative groups as well as at admission vs. discharge in both groups. 


\section{Microbiological diagnosis}

The samples collected under sterile conditions underwent overnight incubation $\left(35^{\circ} \mathrm{C}, 5 \% \mathrm{CO}_{2}\right)$ in $5 \%$ sheep blood agar, chocolate agar and MacConkey agar. A few colonies were taken from the bacteria that were grown on the medium and the saline was spread by dropping the slurry and fixed in flame. Crystal violet dye solution was added to the preparation and left to stand for 1 minute. After washing with distilled water, Lugol solution was added to the preparation and left for 1 minute. The mixture was decolorized for 10-15 seconds by adding distilled water and 95\% ethanol again. The last time it was washed with distilled water and coated with aqueous solution of basic fuchsine and left for 30 seconds. After washing the dye and drying the slide, bacteria were evaluated under the microscope at $100 \times$ for morphological differentiation as well as type identification and antibiogram analysis using the VITEK 2 device (BioMerieux, France).

\section{Statistical analysis}

Statistical analysis was performed using IBM SPSS Statistics for Windows, version 22.0 (IBM Corp., Armonk, NY). The chi-square test was used to analyze categorical data, while the Mann-Whitney $U$ test was used for analysis of numerical data. Change in parameters from admission to discharge was assessed using the Wilcoxon test. Correlation analyses were performed via Spearman's and Pearson's correlation analyses depending on the normality of the distribution. Data were expressed as "mean (standard deviation; SD)", percent (\%) and median (25-75\%) where appropriate. $P<0.05$ was considered statistically significant.

\section{Results}

\section{Comparison of patient characteristics in Gram-positive and Gram-negative pneumonia groups}

Overall, 109 (64.1\%) out of 170 patients were in the $>65$ years age group and males composed $64.1 \%$ of the study population. Gram-positive (Streptococcus pneumoniae in 98.5\%) and Gramnegative (Enterobacteriaceae in $70.0 \%$ ) pathogens were identified in $76.5 \%$ and $23.5 \%$ of isolates, respectively (Table I).

Gram-negative pneumonia compared with Grampositive pneumonia was associated with significantly higher likelihood of being hospitalized at the ICU $(70.0 \%$ vs. $2.3 \%, p<0.001)$, mortality (20.0\% vs. $2.3 \%, p<0.001)$, sepsis $(27.5 \%$ vs. $0.0 \%$, $p<0.001)$, co-morbid bronchiectasis and neuromuscular disease $(7.5 \%$ vs. $0.0 \%, p=0.002$ for each), but lower likelihood of co-morbid asthma (0.0\% vs. $10.0 \%, p=0.037$ ) (Table I).
No significant difference was noted between patients with Gram-positive and Gram-negative pneumonia in terms of age, gender, other comorbidities or length of hospital stay (Table I).

\section{Admission and discharge findings on complete blood count and inflammatory markers in Gram-positive and Gram-negative pneumonia}

Respiration rate $(p<0.001)$, pulse $(p=0.040)$ and body temperature $(p=0.002)$ values were significantly higher in the Gram-negative group as compared with the Gram-positive group (Table II).

Gram-negative pneumonia was associated with significantly lower levels of erythrocytes ( $p<0.001$ for each period), hemoglobin ( $p<0.001$ for each period) and hematocrit ( $p<0.001$ for each period), but with higher levels of MCV ( $p=0.048$ at admission and $p=0.020$ at discharge) as compared with Gram-positive pneumonia both at admission and at discharge (Table II).

Neutrophil to lymphocyte ratio, PLR and PLT/ MPV (at admission or discharge) and CRP levels (at admission) showed no significant difference between Gram-positive and Gram-negative pneumonia. C-reactive protein levels (median (25-75\%) 41.5 (10.8-94.3) vs. 17.1 (7.2-47.3) mg/dl, $p=0.025)$ and CRP/albumin ratio (median (25-75\%) $14.0(2.2-34.4)$ vs. 5.2 (2.1-16.2), $p=$ $0.021)$ at discharge were significantly higher in the Gram-negative than in the Gram-positive group (Table II).

A significant decrease was noted in WBC ( $p<0.001)$ from admission to discharge in Grampositive pneumonia, and a significant decrease in NLR ( $p<0.001$ and $p=0.026$, respectively), CRP ( $p<0.001$ and $p=0.013$, respectively) and CRP/ albumin ratio ( $p=0.021$ and $p=0.037$, respectively) was noted from admission to discharge in both Gram-positive and negative pneumonia groups. Platelet to lymphocyte ratio and PLT/MPV showed no significant change during hospitalization in both groups (Table II).

\section{Correlation of C-reactive protein with other inflammatory parameters}

At admission, CRP levels were correlated positively with WBC $(r=0.47, p=0.009$ and $r=0.36$, $p=0.001$, respectively) and NLR $(r=0.49$, $p=0.006$ and $r=0.40, p<0.001$, respectively) in both Gram-negative and -positive groups, but with PLR ( $r=0.23, p=0.040)$ only in the Gram-positive pneumonia group (Table III).

At discharge, CRP levels were positively correlated with NLR $(r=0.38, p=0.018)$ in the Gramnegative group, but with PLR $(r=0.19, p=0.042)$ in the Gram-positive group (Table III). 
Table I. Comparison of demographic and clinical characteristics of patients in Gram-positive and Gram-negative pneumonia

\begin{tabular}{|c|c|c|c|}
\hline Causative agents & \multicolumn{3}{|c|}{$n(\%)$} \\
\hline Gram-positive & \multicolumn{3}{|c|}{$130(76.5)$} \\
\hline Streptococcus pneumoniae & \multicolumn{3}{|c|}{$128(98.5)$} \\
\hline Gram-negative & \multicolumn{3}{|c|}{$40(23.5)$} \\
\hline Escherichia coli & \multicolumn{3}{|c|}{$3(7.5)$} \\
\hline Enterobacteriaceae & \multicolumn{3}{|c|}{$28(70.0)$} \\
\hline Haemophilus influenzae & \multicolumn{3}{|c|}{$2(5.0)$} \\
\hline Chlamydia & \multicolumn{3}{|c|}{$1(2.5)$} \\
\hline Pseudomonas & \multicolumn{3}{|c|}{$9(22.5)$} \\
\hline Parameter & Gram-positive $(n=130)$ & Gram-negative $(n=40)$ & $P$ value \\
\hline \multicolumn{4}{|l|}{ Age, $n(\%)$} \\
\hline$>65$ years & $70(53.8)$ & $27(67.5)$ & 0.13 \\
\hline$>85$ years & $9(6.9)$ & $3(7.5)$ & 0.90 \\
\hline Gender (male), $n$ (\%) & $79(60.8)$ & $30(75.0)$ & 0.10 \\
\hline \multicolumn{4}{|l|}{ Hospitalization unit, $n(\%)$} \\
\hline General ward & $127(97.7)$ & $12(30.0)$ & \multirow[t]{2}{*}{$<0.001$} \\
\hline ICU & $3(2.3)$ & $28(70.0)$ & \\
\hline Length of stay (days), median (25-75\%) & $6(4-8)$ & $7(4-15)$ & $0.10^{*}$ \\
\hline Mortality, $n(\%)$ & $3(2.3)$ & $8(20.0)$ & $<0.001$ \\
\hline Sepsis, $n(\%)$ & $0(0.0)$ & $11(27.5)$ & $<0.001$ \\
\hline \multicolumn{4}{|l|}{ Co-morbidities, $n(\%)$} \\
\hline COPD & $64(49.2)$ & $23(57.5)$ & 0.36 \\
\hline Diabetes mellitus & $10(7.7)$ & $1(2.5)$ & 0.24 \\
\hline Hypertension & $6(4.6)$ & $0(0.0)$ & 0.17 \\
\hline Congestive heart failure & $15(11.5)$ & $1(2.5)$ & 0.09 \\
\hline Asthma & $13(10.0)$ & $0(0.0)$ & 0.037 \\
\hline Ischemic arterial disease & $4(3.1)$ & $0(0.0)$ & 0.26 \\
\hline Bronchiectasis & $0(0.0)$ & $3(7.5)$ & 0.002 \\
\hline Neuromuscular disease & $0(0.0)$ & $3(7.5)$ & 0.002 \\
\hline Chronic kidney disease & $1(0.8)$ & $0(0.0)$ & 0.58 \\
\hline Immune deficiency & $0(0.0)$ & $1(2.5)$ & 0.07 \\
\hline Collagen diseases & $1(0.8)$ & $0(0.0)$ & 0.58 \\
\hline Cerebrovascular accident & $2(1.5)$ & $1(2.5)$ & 0.69 \\
\hline
\end{tabular}

ICU - intensive care unit, COPD - chronic obstructive pulmonary disease. $\chi^{2}$ test, ${ }^{*}$ Mann-Whitney U test.

CURB-65 scores on admission and associated inflammatory markers

A significantly higher percentage of patients with Gram-negative vs. Gram-positive pneumonia had higher CURB-65 scores (> 4 in 50.0 vs. $0.8 \%$, respectively, $p<0.001$ ) (Table IV).
There was also a non-significant tendency for an increase in median NLR (from 4.1 for CURB-65 score 2 to 12.4 for CURB-65 score 5) and CRP (from 42.2 for CURB-65 score 2 to 96.7 for CURB-65 score 5) levels with increase in CURB-65 scores in the Gram-negative group (Table IV). 


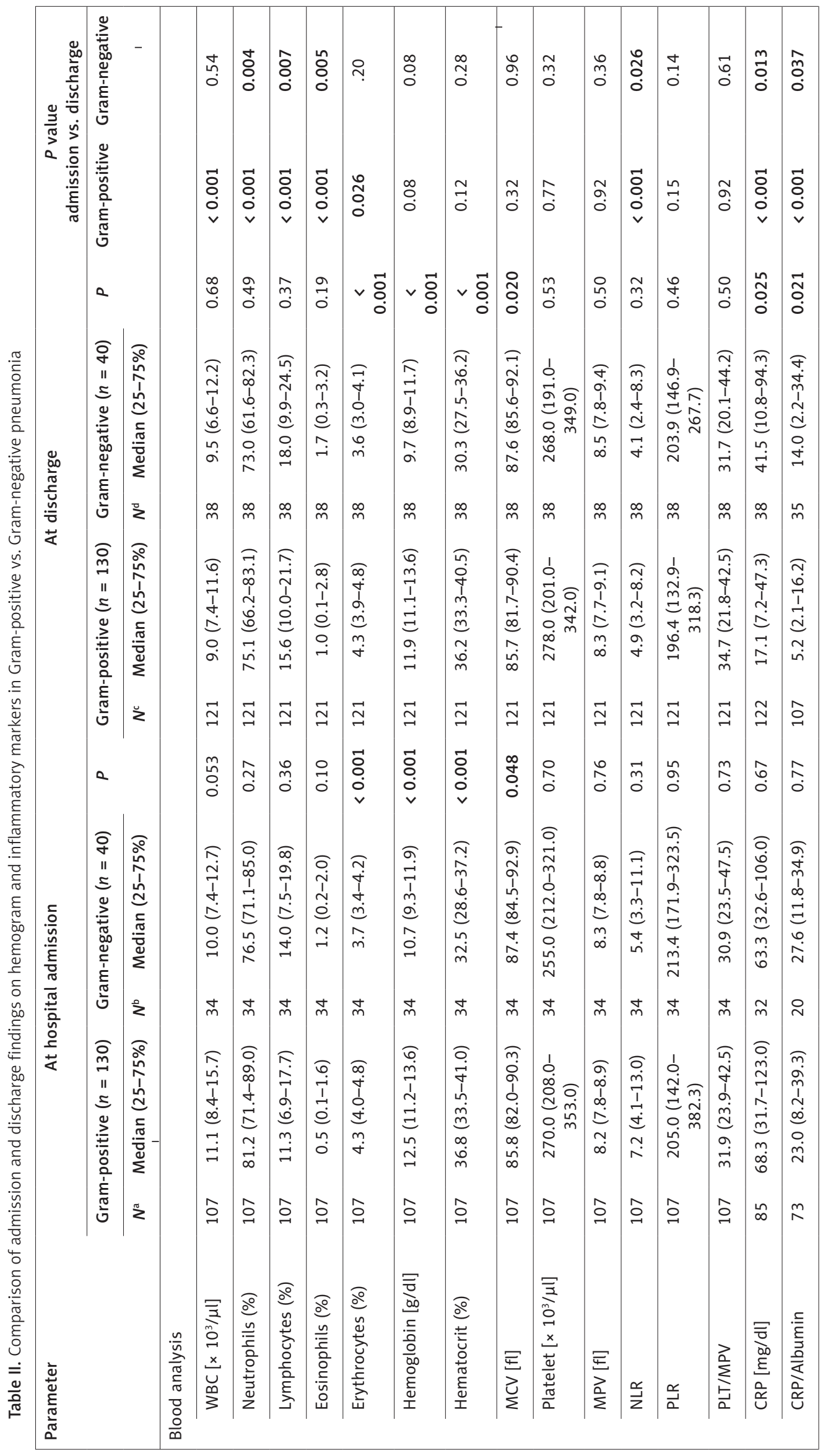




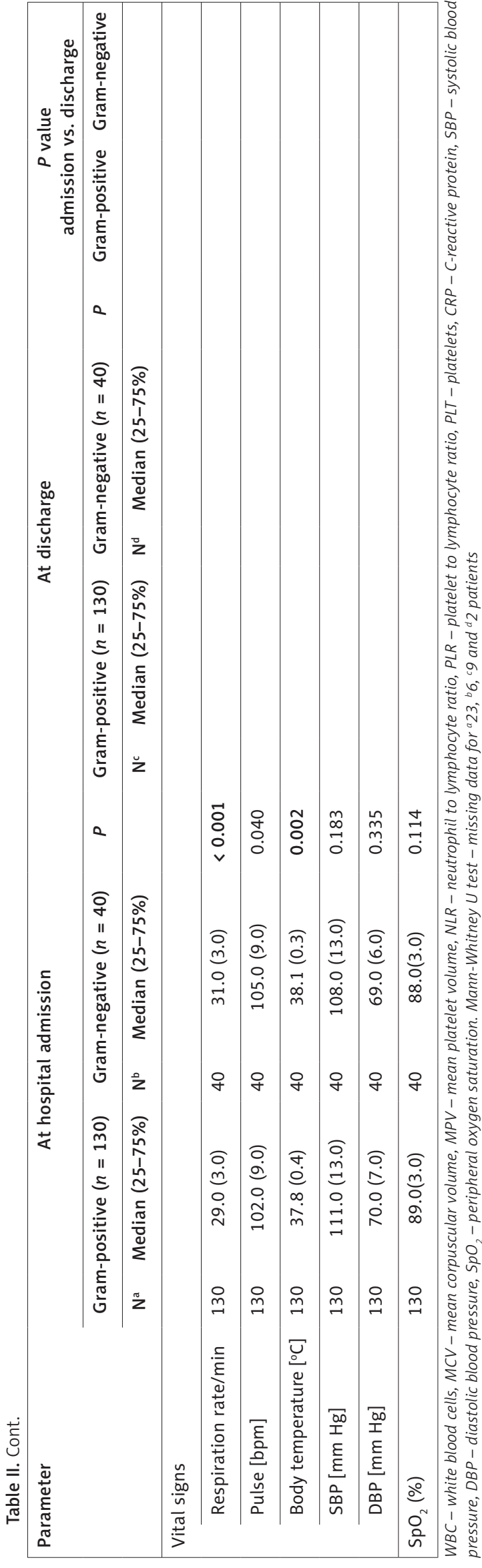

\section{Discussion}

This study explored inflammatory markers (NLR, PLR and PLT/MPV) in differentiation of Gram-positive and Gram-negative CAP in a hospital setting as well as the change in inflammatory markers from admission to discharge with respect to Gram stain results. Our findings revealed a preponderance of Gram-positive pneumonia and poorer clinical outcome in the case of Gram-negative pneumonia with no significant difference between Gram-positive and Gram-negative groups in terms of NLR, PLR and PLT/MPV either at admission or at discharge. C-reactive protein levels and CRP/albumin ratio were also similar at admission between Gram-positive and Gram-negative groups, whereas both were significantly higher in Gram-negative bacterial pneumonia at discharge. From admission to discharge, a significant decrease was noted in NLR, CRP and CRP/albumin ratio in both Gram-negative and Gram-positive groups. CRP levels were positively correlated with NLR (at admission in both groups and at discharge only in the Gram-negative pneumonia group) and with PLR (at admission and discharge only in the Gram-positive pneumonia group).

In our study population, Gram-positive (Streptococcus pneumoniae in 98.5\%) and Gram-negative (Enterobacteriaceae in $70.0 \%$ ) pathogens were identified in $76.5 \%$ and $23.5 \%$ of isolates. This seems consistent with consideration of Streptococcus pneumoniae as the most common causative microorganism of CAP, being reported to comprise $83 \%, 27 \%$ and $28 \%$ of outpatient, inpatients and ICU patient cases in a systematic review of 46 studies [27]. Our findings are also in line with prevalence of etiological diagnoses reported in a prospective study of 178 patients with CAP including pneumococcal pneumonia (52\%) and Gram-negative bacilli pneumonia (18\%) as the two most common pathogens [6].

Given that Streptococcus pneumoniae and Enterobacteriaceae were the major pathogens responsible for Gram-positive and Gram-negative pneumonia in our cohort, poorer clinical outcome with higher likelihood of ICU hospitalization, sepsis and mortality in Gram-negative than in Gram-positive pneumonia seems to be consistent with development of slower resistance of Streptococcus to penicillin [28], but increasing incidence of carbapenem-resistant Enterobacteriaceae challenging the current clinical anti-infective treatment [29] and consideration of Enterobacteriaceae among the emerging multiple drug-resistant pathogens leading to the growing global antimicrobial resistance problem [30]. Indeed, consistent with a less favorable outcome, Gram-negative pneumonia patients in our cohort were discharged with signifi- 
Inflammatory markers in hospitalized patients with bacterial pneumonia: comparison of Gram-positive versus Gram-negative etiology and admission versus discharge findings

Table III. Correlation of C-reactive protein with other inflammatory parameters at admission and at discharge

\begin{tabular}{|c|c|c|c|c|}
\hline \multirow[t]{3}{*}{ Parameter } & \multicolumn{2}{|c|}{ Gram-positive pneumonia $(n=130)$} & \multicolumn{2}{|c|}{ Gram-negative pneumonia $(n=40)$} \\
\hline & At admission & At discharge & At admission & At discharge \\
\hline & CRP & CRP & CRP & CRP \\
\hline \multicolumn{5}{|l|}{ WBC } \\
\hline$r$ & 0.36 & 0.10 & 0.47 & 0.26 \\
\hline$p$ & 0.001 & 0.27 & 0.009 & 0.12 \\
\hline$n$ & 83 & 117 & 34 & 38 \\
\hline \multicolumn{5}{|l|}{ NLR } \\
\hline$r$ & 0.40 & 0.15 & 0.49 & 0.38 \\
\hline$p$ & $<0.001$ & 0.10 & 0.006 & 0.018 \\
\hline$n$ & 83 & 117 & 34 & 38 \\
\hline \multicolumn{5}{|l|}{ PLR } \\
\hline$r$ & 0.23 & 0.19 & 0.0 & 0.47 \\
\hline$p$ & 0.040 & 0.042 & 0.99 & 0.78 \\
\hline$n$ & 83 & 117 & 34 & 38 \\
\hline \multicolumn{5}{|l|}{ PLT/MPV } \\
\hline$r$ & -0.03 & 0.13 & -0.34 & -0.31 \\
\hline$p$ & 0.80 & 0.17 & 0.07 & 0.06 \\
\hline$n$ & 83 & 117 & 32 & 38 \\
\hline
\end{tabular}

$W B C$ - white blood cells, MPV - mean platelet volume, NLR - neutrophil to lymphocyte ratio, PLR - platelet to lymphocyte ratio, $P L T$ - platelet, $C R P-C$-reactive protein. Spearman's and Pearson's correlation analyses. $r$-correlation coefficient.

Table IV. CURB-65 scores on admission and associated inflammatory markers in Gram-positive vs. Gram-negative pneumonia

\begin{tabular}{|c|c|c|c|c|c|c|c|}
\hline \multirow{2}{*}{$\begin{array}{l}\text { CURB-65 } \\
\text { score on } \\
\text { admission }\end{array}$} & $\begin{array}{c}\text { Gram-positive } \\
\quad(n=130)\end{array}$ & $\begin{array}{c}\text { Gram-negative } \\
(n=40)\end{array}$ & & \multicolumn{2}{|r|}{$\begin{array}{c}\text { Gram-positive } \\
\quad(n=130)\end{array}$} & \multicolumn{2}{|r|}{$\begin{array}{l}\text { Gram-negative } \\
(n=40)\end{array}$} \\
\hline & $n(\%)$ & $n(\%)$ & & $n$ & Median (25-75\%) & $n$ & Median (25-75\%) \\
\hline \multirow[t]{2}{*}{2} & $98(75.4)$ & $13(32.5)$ & NLR & 78 & $7.1(4.3-12.1)$ & 11 & $4.1(3.1-8.8)$ \\
\hline & & & CRP $[\mathrm{mg} / \mathrm{dl}]$ & 64 & $69.7(37.5-117.0)$ & 11 & $42.2(29.2-102.0)$ \\
\hline \multirow[t]{2}{*}{3} & $31(23.8)$ & $7(17.5)$ & NLR & 29 & $9.6(3.0-16.5)$ & 6 & $6.2(3.1-7.5)$ \\
\hline & & & CRP $[\mathrm{mg} / \mathrm{dl}]$ & 21 & $50.7(22.8-135.0)$ & 5 & $53.1(35.5-68.4)$ \\
\hline \multirow[t]{2}{*}{4} & $1(0.8)$ & $10(25.0)$ & NLR & 0 & - & 9 & $5.0(4.2-9.0)$ \\
\hline & & & CRP $[\mathrm{mg} / \mathrm{dl}]$ & 0 & - & 10 & $77.6(35.2-143.0)$ \\
\hline \multirow[t]{2}{*}{5} & $0(0.0)$ & $10(25.0)$ & NLR & 0 & - & 8 & $12.4(4.3-15.9)$ \\
\hline & & & CRP $[\mathrm{mg} / \mathrm{dl}]$ & 0 & - & 6 & 96.7 (40.2-109.0) \\
\hline$P$ value & & & & & & & \\
\hline
\end{tabular}

cantly higher CRP and CRP/albumin levels as compared with Gram-positive pneumonia patients, while WBC count significantly decreased from admission to discharge only in the Gram-positive group.

On the basis of lack of a significant difference in NLR, PLR and PLT/MPV both at admission and discharge between Gram-positive and Gram-negative pneumonia groups, our findings indicate no additional benefit of assessing inflammatory markers (NLR, PLR, PLT/MPV, CRP) in differentiation of Gram-negative and Gram-positive pneumonia to aid Gram stain testing in selection of an appropriate therapeutic decision.

Neutrophil to lymphocyte ratio was the only inflammatory marker that showed similarity with 
CRP and CRP/albumin ratio in terms of significant reduction from admission to discharge alongside positive correlation with CRP levels. This seems in agreement with published data on NLR indicating that NLR at the emergency admissions has discriminatory capacity in predicting bacteremia, severity and outcome of CAP with a higher prognostic accuracy as compared with routine markers such as CRP level, neutrophil count and WBC count $[18,19]$. Moreover, both NLR and CRP were reported to be higher in pneumonia patients with complications as compared with those without complications [4].

Although no significant difference was noted between Gram-positive and Gram-negative groups in terms of NLR in our cohort, the tendency for higher NLR in the Gram-positive group seems to support the data from a past study in pneumonia patients indicating the association of Streptococcus pneumoniae with the highest frequency of increased NLR values as compared with other pathogens [18]. Nonetheless, while this association was considered to be related to the severity of disease in patients with pneumococcal pneumonia as reflected in increased duration of hospitalization, ICU admittance and mortality [18], our findings revealed a less favorable outcome in terms of ICU admittance sepsis and mortality in the Gram-negative pneumonia group. CURB-65 is amongst the frequently used scoring methods for hospitalization and treatment decisions in CAP patients in Turkey [31]. Notably, having higher CURB-65 scores on admission was also more likely in patients with Gram-negative pneumonia along with a tendency for increase in NLR and CRP levels with increase in CURB-65 scores. This seems to be consistent with poor prognosis in patients with Gram-negative pneumonia in the current study.

Management of CAP remains challenging in relation to difficulties in diagnosis of the causative pathogen, and subsequent identification of prognosis and severity of disease [17]. Our study failed to show a statistically significant difference between Gram-negative and Gram-positive groups in terms of inflammatory markers at admission or discharge and thus to help differentiation between bacterial etiology. This seems in accordance with the recommendation of sputum and blood culture in these cases by the Guidelines on Community-Acquired Pneumonia 2019 [17].

Nonetheless, given that both NLR and PLR were correlated positively with CRP levels at admission in Gram-positive pneumonia, our findings seem to indicate higher likelihood of NLR and PLR having an additional benefit in evaluation of the inflammatory process and treatment response, as a CRP substitute, in Gram-positive pneumonia rather than in Gram-negative pneumonia in hospitalized patients with CAP.

Certain limitations to this study should be considered. First, due to the retrospective single-center design, establishing the temporality between cause and effect as well as generalizing our findings to the overall CAP population seems difficult. Second, lack of data on type of antibiotics prescribed and resistance profiles of causative pathogens is another limitation which otherwise would extend the knowledge achieved in the current study. Nevertheless, despite these certain limitations, given the paucity of solid information available in this area, our findings represent a valuable contribution to the literature.

In conclusion, this retrospective study of hospitalized CAP patients revealed no significant role of NLR, PLR, PLT/MPV or CRP in differential diagnosis of Gram-negative versus Gram-positive etiology and thus no additional benefit of these markers in faster implementation of appropriate treatment in accordance with the Gram stain. The significant decrease in NLR from admission to discharge alongside the positive correlation with CRP at admission seems to emphasize the potential role of NLR in evaluation of treatment response, as a substitute of CRP, particularly in those with Gram-positive CAP. Larger scale prospective studies with more comprehensive microbiological analysis are needed to determine the additional benefit of inflammatory markers in aiding microbiology-based etiological identification for earlier recognition of the responsible pathogen and prompter implementation for monitoring the treatment success and clinical outcome.

\section{Conflict of interest}

The authors declare no conflict of interest.

\section{References}

1. Lim WS, Smith DL, Wise MP, et al.; British Thoracic Society. British Thoracic Society community acquired pneumonia guideline and the NICE pneumonia guideline: how they fit together. Thorax 2015; 70: 698-700.

2. Musher DM, Thorner AR. Community-acquired pneumonia. N Engl J Med 2014; 371: 1619-28.

3. Remington LT, Sligl W. Community-acquired pneumonia. Curr Opin Pulm Med 2014; 20: 215-24.

4. Bekdas M, Goksugur SB, Sarac EG, et al. Neutrophil/ lymphocyte and C-reactive protein/mean platelet volume ratios in differentiating between viral and bacterial pneumonias and diagnosing early complications in children. Saudi Med J 2014; 35: 442-7.

5. Yan ST, Sun LC, Lian R, et al. Diagnostic and predictive values of procalcitonin in bloodstream infections for nosocomial pneumonia. J Crit Care 2018; 44: 424-9.

6. Anevlavis S, Petroglou N, Tzavaras A, et al. A prospective study of the diagnostic utility of sputum Gram stain in pneumonia. J Infect 2009; 59: 83-9. 
7. Woodhead M, Blasi F, Ewig S, et al. Guidelines for the management of adult lower respiratory tract infections. Clin Microbiol Infect 2011; 17: 1-59.

8. Rhodes A, Evans LE, Alhazzani W, et al. Surviving sepsis campaign: international guidelines for management of sepsis and septic shock: 2016. Intensive Care Med 2017; 43: 304-77.

9. Broughton WA, Middleton III RM, Kirkpatrick MB, et al. Bronchoscopic protected specimen brush and bronchoalveolar lavage in the diagnosis of bacterial pneumonia. Infect Dis Clin North Am 1991; 5: 437-52.

10. Reed WW, Byrd GS, Gates Jr RH, et al. Sputum Gram's stain in community-acquired pneumococcal pneumonia: a meta-analysis. West J Med 1996; 165: 197-204.

11. Leven M, Goosens H. Relevance of nucleic acid amplification techniques for diagnosis of respiratory tract infections in the clinical laboratory. Clin Microbiol Rev 1997; 10: 242-56.

12. Boerner DF, Zwadyk P. The value of the sputum Gram's stain in community-acquired pneumonia. J Am Med Assoc 1982; 247: 642-5.

13. Kalin M, Lindberg AA, Tunewall G. Etiological diagnosis of bacterial pneumonia by Gram stain and quantitative culture of expectorates. Scand J Infect Dis 1983; 15: 153-60.

14. Christ-Crain M, Muller B. Biomarkers in respiratory tract infections: diagnostic guides to antibiotic prescription, prognostic markers and mediators. Eur Respir J 2007; 30: 556-73.

15. Schuetz P, Christ-Crain M, Muller B. Biomarkers to improve diagnostic and prognostic accuracy in systemic infections. Curr Opin Crit Care 2007; 13: 578-85.

16. Kopterides P, Siempos, II, Tsangaris I, et al. Procalcitonin-guided algorithms of antibiotic therapy in the intensive care unit: a systematic review and meta-analysis of randomized controlled trials. Crit Care Med 2010; 38: 2229-41.

17. Metlay JP, Waterer GW, Long AC, et al. Diagnosis and treatment of adults with community-acquired pneumonia. An official clinical practice guideline of the American Thoracic Society and Infectious Diseases Society of America. Am J Respir Crit Care Med 2019; 200: e45-e67.

18. de Jager CP, Wever PC, Gemen EF, et al. The neutrophillymphocyte count ratio in patients with communityacquired pneumonia. PLoS One 2012; 7: e46561.

19. de Jager CP, van Wijk PT, Mathoera RB, et al. Lymphocytopenia and neutrophil-lymphocyte count ratio predict bacteremia better than conventional infection markers in an emergency care unit. Crit Care 2010; 14: 192.

20. Martinez R, Menendez R, Reyes S, et al. Factors associated with inflammatory cytokine patterns in communityacquired pneumonia. Eur Respir J 2011; 37: 393-9.

21. Chalupa P, Beran O, Herwald H, et al. Evaluation of potential biomarkers for the discrimination of bacterial and viral infections. Infection 2011; 39: 411-7.

22. Adams NG. Diagnostic use of C-reactive protein in bacteraemic emergency department patients. Emerg Med Australas 2005; 17: 371-5.

23. Yao C, Liu X, Tang Z. Prognostic role of neutrophil-lymphocyte ratio and platelet-lymphocyte ratio for hospital mortality in patients with AECOPD. Int J Chron Obstruct Pulmon Dis 2017; 12: 2285-90.

24. Curbelo J, Luquero Bueno $\mathrm{S}$, et al. Inflammation biomarkers in blood as mortality predictors in communityacquired pneumonia admitted patients: importance of comparison with neutrophil count percentage or neutrophil-lymphocyte ratio. PLoS One 2017; 12: e0173947.
25. Yang T, Wan $\mathrm{C}$, Wang $\mathrm{H}$, et al. The prognostic and riskstratified value of neutrophil-lymphocyte count ratio in Chinese patients with community-acquired pneumonia. Eur J Inflamm 2017; 15: 22-7.

26. Karataş MB, Ipek G, Onuk T, et al. Assessment of prognostic value of neutrophil to lymphocyte ratio and platelet to lymphocyte ratio in patients with pulmonary embolism. Acta Cardiol Sin 2016; 32: 313-20.

27. Welte T, Torres A, Nathwani D. Clinical and economic burden of community acquired pneumonia among adults in Europe. Thorax 2012; 67: 71-9.

28. Amyes SG. Enterococci and streptococci. Int J Antimicrob Agents 2007; 29: 43-52.

29. Pang F, Jia XQ, Zhao QG, et al. Factors associated to prevalence and treatment of carbapenem-resistant Enterobacteriaceae infections: a seven years retrospective study in three tertiary care hospitals. Ann Clin Microbiol Antimicrob 2018; 17: 13.

30. MacGowan A, Macnaughton E. Antibiotic resistance. Medicine 2017; 45: 622-8.

31. Ozyürek BA, Erturk A, Aydemir Y, et al. The approach to community-acquired pneumonia: a survey study. Eurasian J Pulmonol 2018; 20: 93-8. 